\title{
On creep-fatigue endurance of TIG-dressed weldments using the linear matching method
}

\author{
Yevgen Gorash*, Haofeng Chen
}

Department of Mechanical E Aerospace Engineering, University of Strathclyde, James Weir Building, 75 Montrose Street, Glasgow G1 1XJ, UK

\begin{abstract}
This paper is devoted to parametric study on creep-fatigue endurance of the steel type $316 \mathrm{~N}(\mathrm{~L})$ weldments at $550^{\circ} \mathrm{C}$ identified as type 3 according to R5 Vol. 2/3 procedure classification. The study is implemented using a direct method known as the Linear Matching Method (LMM) and based upon the creep-fatigue evaluation procedure considering time fraction rule for creep-damage assessment. Seven configurations of the weldment, characterised by particular values of a geometrical parameter $\rho$, are proposed. Parameter $\rho$, which represents different grades of TIG dressing, is a ratio between the radius of the fillet of the remelted metal on a weld toe and the thickness of welded plates. For each configuration, the total number of cycles to failure $N^{\star}$ in creep-fatigue conditions is assessed numerically for different loading cases defined by normalised bending moment $\tilde{M}$ and dwell period $\Delta t$. The obtained set of $N^{\star}$ is extrapolated by the analytic function dependent on $\tilde{M}, \Delta t$ and parameter $\rho$. Proposed function for $N^{\star}$ shows good agreement with numerical results obtained by the LMM. Therefore, it is used for the identification of Fatigue Strength Reduction Factors (FSRFs) effected by creep, which are intended for design purposes, and dependent on $\Delta t$ and $\rho$.
\end{abstract}

Keywords: Creep, Damage, Finite element analysis, FSRF, Low-cycle fatigue, Type 316 steel, Weldment

\section{Introduction}

According to industrial experience, during the service life of welded structures subjected to cyclic loading at high temperature, welded joints are usually considered as the critical locations of potential creep-fatigue failure. This is caused by higher stress concentration, altered and non-uniform material properties of weldments compared to the parent material of the entire structure. Therefore, creep and fatigue characteristics of welded joints are of a priority importance for long-term integrity assessments and design of welded structures. There were many attempts to develop analytical tools $[1,2,3,4]$ to estimate long-term strength of welded joints under variable loading. However, residual life assessments are frequently complicated and inaccurate because of complex material microstructure and too many parameters affecting the strength of welded joints. They include technological parameters of welding process and post-weld heat treatment, accuracy of modelling of weldment material microstructure, influence of residual stresses and distortions, geometrical parameters of the shape of the weld profile and non-welded root gaps, parameters of service conditions such as temperature, mechanical loading and dwell period. In view of the complexity of a unified model development for the assessment of creep-fatigue strength, there are a limited number of existing analytical approaches, but none of which are able to account for all of weldment parameters mentioned above. Thus, long-term strength of weldments is a wide research area, which requires some unified integral approach able to improve the life prediction capability for welded joints. The most comprehensive overviews of studies devoted to investigation of influence of various parameters on fatigue life of welded joints are presented in [1, 2, 3, 4].

It should be noted that the influence of creep on residual life is not investigated in these works. However, the recent edition of the British Standard 7910 [5] presents some basic guideline for the assessment of flaws in austenitic and ferritic weldments in creep-fatigue conditions. Moreover, BS 7910 [5] includes methods for assessment of fracture,

${ }^{*}$ Corresponding author. Tel.: +44 141 5524400; Fax: +44 1415520775.

Email addresses: yevgen.gorash@strath.ac.uk (Yevgen Gorash), haofeng.chen@strath.ac.uk (Haofeng Chen) 
fatigue crack growth, creep crack growth and "other modes" of failure, e.g. stress corrosion and buckling, of structures with flaws.

This paper presents a further extension of a recently developed approach [6], which includes a creep-fatigue evaluation procedure considering time fraction rule for creep-damage assessment and a recent revision of the Linear Matching Method (LMM) to perform a cyclic creep assessment [7]. The applicability of this approach to a creep-fatigue analysis was verified in [6] by the comparison of FEA/LMM predictions for an AISI type 316N(L) steel cruciform weldment at $550^{\circ} \mathrm{C}$ with experiments by Bretherton et al. $[8,9,10,11]$ with the overall objective of identifying fatigue strength reduction factors (FSRF) of austenitic weldments for further design applications. An overview of previous modelling studies devoted to analysis and simulation of these experiments $[8,9,10,11]$ is given in [6]. Generally they investigated an accuracy of residual life assessments according to R5 creep-fatigue crack initiation procedure [12] and its more recent revisions and potential improvements.

Effective and fast modelling of structural components with complex microstructure and material behaviour such as weldments under high-temperature and variable loading conditions can be implemented by the application of FEA with direct analysis methods, which calculate the stabilised cyclic response of structures with far less computational effort than full step-by-step analysis. The most practical among these methods are Direct Cyclic Analysis [13, 14] and the LMM framework $[15,16]$. It should be noted that the predecessor of the LMM is "the method of variable coefficients of elasticity" formulated by Birger [17]. As mentioned in [18], it was applied to the solutions of the non-linear equations of deformation theory in the case of hardening using successive approximations.

The LMM is distinguished from the other simplified methods by ensuring that both the equilibrium and compatibility are satisfied at each stage $[15,16,19,20]$. In addition to the shakedown analysis method [19], the LMM has been extended beyond the range of most other direct methods by including the evaluation of the ratchet limit [15, 16, 20] and steady-state cyclic behaviour with creep-fatigue interaction [21, 22]. The LMM ABAQUS user subroutines [23] have been consolidated by the R5 Procedure [12] research programme of EDF Energy to the commercial standard, and are counted to be the method most amenable to practical engineering applications involving complicated thermomechanical load history [16, 20]. Following this, the LMM was much improved both theoretically and numerically [7] to include more accurate predictions of the stabilised cyclic response of a structure under creep-fatigue conditions. This, in turn, allowed more accurate assessments of the resulting cyclic and residual stresses, creep strain, plastic strain range, ratchet strain and elastic follow-up factor. Finally, to aid wider adoption of the LMM as an analysis tool for industry, the development of an Abaqus/CAE plug-in with GUI has been started [24]. For this purpose, the UMAT subroutine code has been updated [24] to allow use of multi-processors for the FE-calculations of shakedown and ratchet limits.

The parametric study presented in this paper is based on the research outcomes given in prior work [6] successfully validated by matching the basic experiments $[8,9,10,11]$. These outcomes briefly include: 1) more realistic modelling of a material behaviour of the weld regions including LCF and creep endurance; 2) a creep-fatigue evaluation procedure considering time fraction rule for creep-damage assessment and a non-linear creep-fatigue interaction diagram; 3) application of the recent revision of the LMM outlined in [7]. As a result, the approach proposed in [6] provides the most accurate numerical prediction of the experiments $[8,9,10,11]$ with less conservatism when compared to previous works, particularly to [22].

Another outcome of the work [6] is the formulation of an analytical function for the total number of cycles to failure $N^{\star}$ in creep-fatigue conditions, which is dependent on normalised bending moment $\tilde{M}$ and dwell period $\Delta t$. This function $N^{\star}(\tilde{M}, \Delta t)$ matches the LMM predictions with reasonable accuracy and is used for the investigation of $\Delta t$ influence on the FSRF. Therefore, the effect of creep on long-term strength is taken into account for type 2 dressed weldments according to the classification in R5 Vol. 2/3 Procedure [12].

Apart from the effect of operating parameters $(\tilde{M}$ and $\Delta t)$, the influence of a weld profile geometry on creep-fatigue strength is investigated within several parametric studies. The introduction of geometrical parameters (angles $\alpha$ and $\beta$ ) into the function $N^{\star}(\tilde{M}, \Delta t)$ allowed the calculation of the FSRF as a continuous function able to cover a variety of weld profile geometries for types 1 and 2 including dressed, as-welded and intermediate configurations as reported in [25]. Thus, exactly the same assessment approach $[6,25]$ is used in the current work and is applied to parametric study of the type 3 weldment geometry in order to assess the effect on the predicted life and the FSRF influenced by creep and geometric parameters variation. 


\section{Parametric models of weldments}

\subsection{Geometrical relations}

Referring to [1], generally creep-fatigue test results of weldment specimens contain various levels of scatter, which is usually caused by geometric and processing variations such as part fit-up, weld gap, variation in feed rates, travel rates, weld angles, etc. This scatter complicates the interpretation of test results, and often makes it nearly impossible to differentiate the effects of geometry, material non-uniformity, residual stress and other factors. It has been indicated [1] that one of the most critical factors affecting the creep-fatigue life of a welded joint is the consistency of the crosssectional weld geometry. The simplified weld profile is usually characterised by the following geometric parameters [1]: plate thickness, effective weld throat thickness, weld leg length, weld throat angle, and weld toe radius. Usually, the weld profile is assumed to be circular for type 1, circular or triangular for type 2, and triangular for type 3 weldments with fillets on toes connecting with parent plates. A vast quantity of researches reviewed in [1, 2, 3, 4] has been devoted to investigation of effects produced by these parameters on residual life.

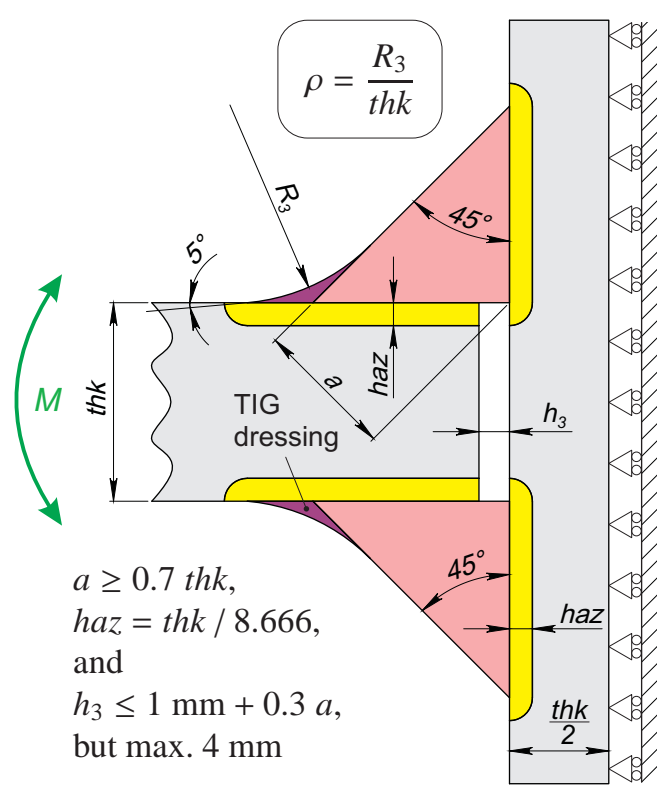

Figure 1: Designations of parameters for type 3 weldment profile and applied bending moment, according to [26, 27]
Table 1: Geometrical configurations of the type 3 weldment

\begin{tabular}{c|ccccccc}
\hline \hline Conf. & 1 & 2 & 3 & 4 & 5 & 6 & 7 \\
\hline$\rho$ & 2.0 & 1.5 & 1.0 & 0.5 & 0.2 & 0.1 & 0 \\
\hline \hline
\end{tabular}

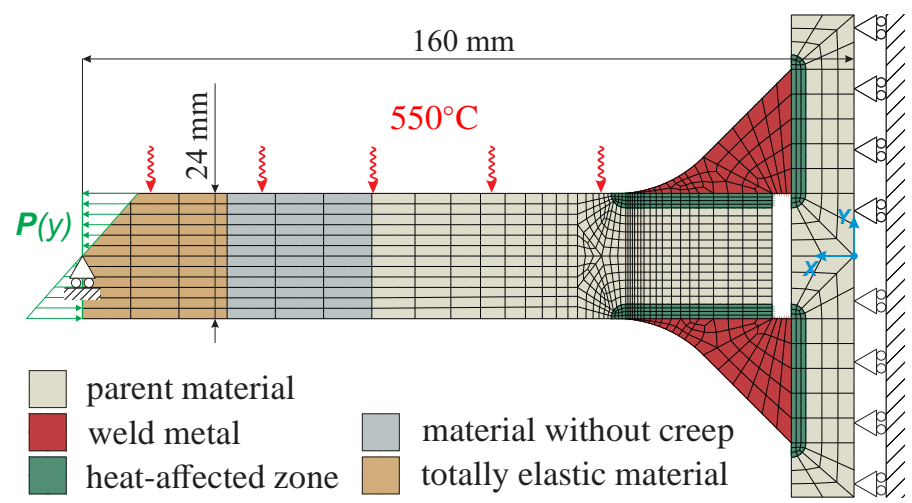

Figure 2: FE-mesh for type 3 weldment with designation of different materials, boundary conditions and mechanical loading

In the present study, the geometry of the weld profile for type 3 weldment is more completely specified in order to investigate its as-welded, dressed and intermediate configurations. The basis of the parametric model for type 3 weldment shown in Fig. 1 are the sketches of weld profiles and corresponding regulations from British Standards $[26,27]$ for the weldment, which contains a root gap between the parts to be joined. The type 3 weldment specimen contains 2 symmetric double-sided T-butt cruciform mitre-fillet welds. The parent material for the manufacturing of all specimens are continuous plates of width $w=200 \mathrm{~mm}$ and thickness $t h k=26 \mathrm{~mm}$ made of the steel type AISI $316 \mathrm{~N}(\mathrm{~L})$. The typical division of the weld into three regions is adopted here analogically to [6, 25] including: parent material, weld metal and heat-affected zone (HAZ). It should be noted that the HAZ thickness is assumed to be $3 \mathrm{~mm}$ based on the geometry given in [8].

Since the geometry of type 3 weldment profile due to mitre fillet is much simpler compared to the geometry of type 1 and 2 weldments, there are only a few parameters governing this type of geometry. The form of type 3 weld is a isosceles triangle with right angle, as shown in Fig. 1. It is characterised by the weld throat $a$, which should be ( $a \geq 0.7 \mathrm{thk}$ ) according to the standard [26]. The gap between the welded parts $h_{3}$ should satisfy the requirement $\left(h_{3} \leq\{1+0.3 a\} \mathrm{mm}\right)$, but it shouldn't exceed $4 \mathrm{~mm}$ according to the standard [27]. 
The fatigue performance of the original type 3 weld profile is quite poor due to significant stress concentration in the weld toe caused by inconsistency of weld profile in $45^{\circ}$. Moreover, the gap between the welded parts decreases the effective cross-section limiting it to the only area of weld metal. For the purpose of the fatigue life improvement, different post weld treatment techniques are applied to the weld toe, as a potential location of failure. TIG (tungsten-arc inert-gas) dressing was found in [28] to be the best suited post weld treatment for implementation in mass production compared to burr grinding and ultrasonic impact treatment, because of the large improvement observed in the experiments (up to $40 \%$ increase in fatigue strength). Therefore, $R_{3}$ in Fig. 1 is the radius of fillet produced by TIG dressing on the weld toe. The angle of discrepancy for the tangency condition between TIG weld metal and patent plate is $5^{\circ}$, since it is a minimum allowable angle for a finite element in order not to be distorted.

Types 1 and 2 weld profiles were governed in [25] by two geometrical parameters (angles $\alpha$ and $\beta$ ) to characterise different scales of weldment dressing by grinding using five configurations. Analogically, in order to reduce the computational costs, seven configurations of weld profile, listed in Table 1, are chosen for parametric study of the type 3 weldment. The different scales of TIG dressing are characterised by the parameter ratio between fillet radius and plate thickness: $\rho=R_{3} /$ thk. Configuration no. 1 with $\rho=2.0$ corresponds to perfectly dressed condition, while configuration no. 7 with $\rho \rightarrow 0$ corresponds to as-welded condition.

\subsection{Finite element models}

The FE-mesh for the 2D symmetric model of type 3 weldment is shown in Fig. 2 assuming plane strain conditions. Analogically to [6, 25], the FE-model includes 5 separate areas with different material properties: 1) parent material, 2) HAZ, 3) weld metal, 4) material without creep, 5) totally elastic material. Introduction of 2 additional material types (material without creep and totally elastic material) representing reduced sets of parent material properties in the location of bending moment application avoids excessive stress concentrations. The FE-model is meshed with ABAQUS element type CPE8R: 8-node biquadratic plane strain quadrilaterals with reduced integration. The FE-mesh for type 3 weldwent contains the range of elements from 1008 for Conf. 1 to 908 for Conf. 7 ( $\rho \rightarrow 0$ ) respectively.

The basic testing $[8,9,10,11]$ was performed at $550 \pm 3^{\circ} \mathrm{C}$ under fully-reversed 4-point bending with total strain ranges $\Delta \varepsilon_{\text {tot }}$ of $0.25,0.3,0.4,0.6$ or $1.0 \%$ in the parent plate and hold periods $\Delta t$ of 0,1 or 5 hours using a strain rate of $0.03 \% / \mathrm{s}$. For the purpose of shakedown and creep analysis using LMM, the conversion from strain-controlled test conditions to force-controlled loading in the simulations using bending moment $M$ has been explained in [6].

Another effective analysis technique, successfully employed in [6], was to apply the bending moment $M$ through the linear distribution of normal pressure $P$ over the section of the plate as illustrated in Fig. 2 with the area moment of inertia in regard to horizontal axis $X: I_{X}=w t h k^{3} / 12$, where the width of plate $w=200 \mathrm{~mm}$ and the thickness of plate $t h k=26 \mathrm{~mm}$. Therefore, the normal pressure is expressed in terms of applied bending moment $M$ and vertical coordinate $y$ of plate section assuming the coordinate origin in the mid-surface: $P(y)=M y / I_{X}$.

Mechanical properties of the materials composing cruciform weldment manufactured of the steel AISI type $316 \mathrm{~N}(\mathrm{~L})$ include the following material behaviour models at $550^{\circ} \mathrm{C}$ [6]:

1. Ramberg-Osgood (R-O) model for the plastic and total strains under saturated cyclic conditions:

$$
\frac{\Delta \varepsilon_{\mathrm{tot}}}{2}=\frac{\Delta \sigma}{2 \bar{E}}+\left(\frac{\Delta \sigma}{2 B}\right)^{1 / \beta} \text { with } \bar{E}=\frac{3 E}{2(1+v)} ;
$$

2. S-N diagrams for the number of cycles to failure caused by pure low-cycle fatigue (LCF):

$$
\log \left(\Delta \varepsilon_{\mathrm{tot}}\right)=m_{0}+m_{1} \log \left(N^{*}\right)+m_{2} \log \left(N^{*}\right)^{2}
$$

3. Power-law model in "time hardening" form for creep strains during primary creep stage:

$$
\dot{\varepsilon}^{\mathrm{cr}}=A \sigma^{n} t^{m} \quad \text { or } \quad \varepsilon^{\mathrm{cr}}=[A /(m+1)] \sigma^{n} t^{m+1}
$$

4. Reverse power-law relation for the time to creep rupture caused by creep relaxation during dwells:

$$
t^{*}=B \sigma^{-k}
$$


5. Non-linear diagram for creep-fatigue damage interaction [29] to define total number of cycles to failure:

$$
\frac{\omega_{\mathrm{f}}}{1-\omega_{\mathrm{cr}}}+\frac{\omega_{\mathrm{cr}}}{1-\omega_{\mathrm{f}}}=1
$$

All the material constants for parent, weld and $\mathrm{HAZ}$ materials at $550^{\circ} \mathrm{C}$ required for cyclic plasticity model $(B, \beta$, $E, v)$ in Eq. (1), LCF failure estimation $\left(m_{0}, m_{1}, m_{2}\right)$ in Eq. (2), creep strain model $(A, n, m)$ in Eq. (3), creep failure estimation $(B, k)$ in Eq. (4) are reported in [6].

\section{Plastic bending of plates}

\subsection{Solution with Ramberg-Osgood model}

The R-O model (1) has the advantage that it can be used to accurately represent the stress-strain curves of metals that harden with plastic deformation, showing a smooth elastic-plastic transition at high temperatures. It establishes the relation between the total strain range $\Delta \varepsilon_{\text {tot }}$ and the equivalent stress range $\Delta \sigma$ in MPa using the material constants $B$ and $\beta$ to describe plastic strain and the Young's modulus $E$ in MPa and the Poisson's ratio $v$ to describe elastic strain.

Although this relationship (1) is not explicitly solvable for stress range $\Delta \sigma$, an approximate solution for $\Delta \sigma$ can be found using following recursive formulation:

$$
\frac{\Delta \sigma_{n+1}}{2}=B\left(\frac{\Delta \varepsilon_{\mathrm{tot}}}{2}-\frac{\Delta \sigma_{n}}{2 \bar{E}}\right)^{\beta} \quad \text { with } \quad n \geq 3,
$$

where the initial iteration is defined as

$$
\frac{\Delta \sigma_{0}}{2}=\left(\frac{\Delta \varepsilon_{\mathrm{tot}}}{2}\right)^{\beta}
$$

For the case of plastic bending of a plate with a rectangular cross-section, i.e. as was used in the experimental studies implemented by Bretherton et al. [8, 9, 10, 11], it is possible to formulate an analytic relation using the R-O material model for the applied bending moment $M$ as proposed in [30]:

$$
M=\frac{2 w \sigma_{\text {eop }}}{3} \frac{t h k^{2}}{4} \frac{1+\frac{3 \beta+3}{2 \beta+1} \tilde{\varepsilon}+\frac{3}{\beta+2} \tilde{\varepsilon}^{2}}{(1+\tilde{\varepsilon})^{2}},
$$

where the maximum normal stress over a cross-section or edge-of-plate stress $\sigma_{\text {eop }}$ is defined based upon the plane strain assumption using equivalent stress $\sigma$

$$
\sigma_{\text {eop }}=2 \sigma / \sqrt{3}=\Delta \sigma / \sqrt{3}
$$

and the ratio between plastic and elastic strains is

$$
\tilde{\varepsilon}=\frac{\varepsilon_{\mathrm{pl}}}{\varepsilon_{\mathrm{el}}}=\left(\frac{\Delta \sigma}{2 B}\right)^{1 / \beta} \frac{2 \bar{E}}{\Delta \sigma} .
$$

Other parameters of relation (8) include the material constants of the $\mathrm{R}-\mathrm{O}$ model $(\beta, B, \bar{E})$ and the geometric parameters of a plate $(t h k$ and $w)$. For the case of reverse bending tests of cruciform weldments at $550^{\circ} \mathrm{C}$ implemented by Bretherton et al. $[8,9,10,11]$, the total strain range $\Delta \varepsilon_{\text {tot }}$ in outer fibre of parent material plate remote from weld was controlled to correspond to one of the required values. Knowledge of the stabilised cycle parent material properties of the steel AISI type $316 \mathrm{~N}(\mathrm{~L})$ described by the R-O model (1) reported in Table 1 of [6] and geometric parameters of specimen $(t h k=26 \mathrm{~mm}$ and $w=200 \mathrm{~mm})$ allows the calculation of the values of bending moments applied in experiments $[8,9,10,11]$ during the period of saturated cyclic response, as reported in Table 2.

Referring to [30], Eq. (8) gives a smooth variation of moment with strain, which could be derived analytically employing recursive formulas (6) and (7) for $\Delta \sigma$ dependent on $\Delta \varepsilon_{\text {tot }}$. Applying the recursive approach, the dependence 
of total strain range $\Delta \varepsilon_{\text {tot }}$ on applied moment $M$ could be obtained. Firstly, Eq. (8) is inverted to recursive formula taking into account Eq. (9):

$$
\begin{aligned}
& \frac{\Delta \sigma_{n+1}}{2}=\frac{M}{\frac{4 w}{3 \sqrt{3}} \frac{t h k^{2}}{4} \frac{1+\frac{3 \beta+3}{2 \beta+1} \tilde{\varepsilon}_{n}+\frac{3}{\beta+2} \tilde{\varepsilon}_{n}^{2}}{\left(1+\tilde{\varepsilon}_{n}\right)^{2}}} \\
& \text { with } \tilde{\varepsilon}_{n}=\left(\frac{\Delta \sigma_{n}}{2 B}\right)^{1 / \beta} \frac{2 \bar{E}}{\Delta \sigma_{n}} \quad \text { and } n \geq 3,
\end{aligned}
$$

where the initial iteration is defined as

$$
\frac{\Delta \sigma_{0}}{2}=\frac{M}{\frac{2 w}{3} \frac{2}{\sqrt{3}}\left(\frac{t h k}{2}\right)^{2} \frac{3}{\beta+2}}
$$

Secondly, the conventional formulation of the R-O model (1) is applied to evaluate the total strain range $\Delta \varepsilon_{\text {tot }}$ corresponding to the equivalent stress range obtained in Eqs (11) and (12). Such a useful relation for $\Delta \varepsilon_{\text {tot }}(M)$ allows the estimation of an important control parameter of the LCF experiments, when the geometry of specimen is known and plastic deformation of a material is comprehensively described by the R-O model. Figure 3 illustrates the application of both approaches (direct by Eqs (6-10) and inverted by Eqs $(1,11,12)$ ) to the parent material plate used in the experiments $[8,9,10,11]$ with particular dimensions of cross-section $(t h k=26 \mathrm{~mm}$ and $w=200 \mathrm{~mm})$ and particular material properties described by the R-O model $(E=160 \mathrm{GPa}, v=0.3, B=1741.96 \mathrm{MPa}, \beta=0.2996)$.

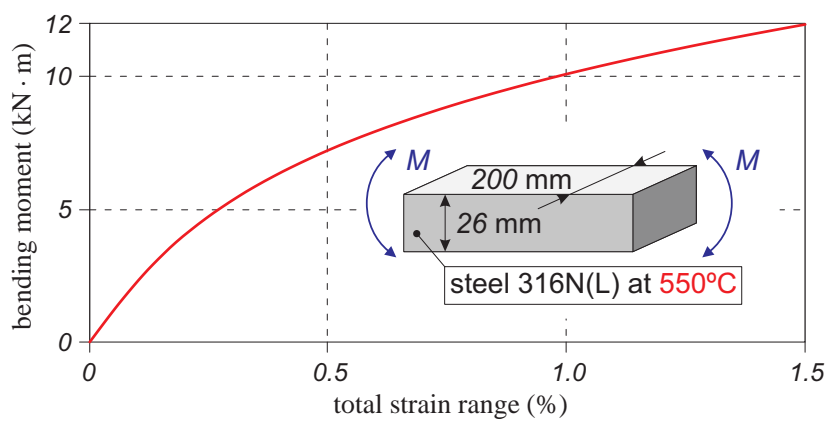

Figure 3: Curve presenting $M$ vs. $\Delta \varepsilon_{\text {tot }}$ relationship for a parent plate with particular cross-section and described by particular R-O model material constants
Table 2: Bending moments $M$ obtained by Eqs (6-10) corresponding to the strain ranges $\Delta \varepsilon_{\text {tot }}$ from experiments $[8,9,10,11]$

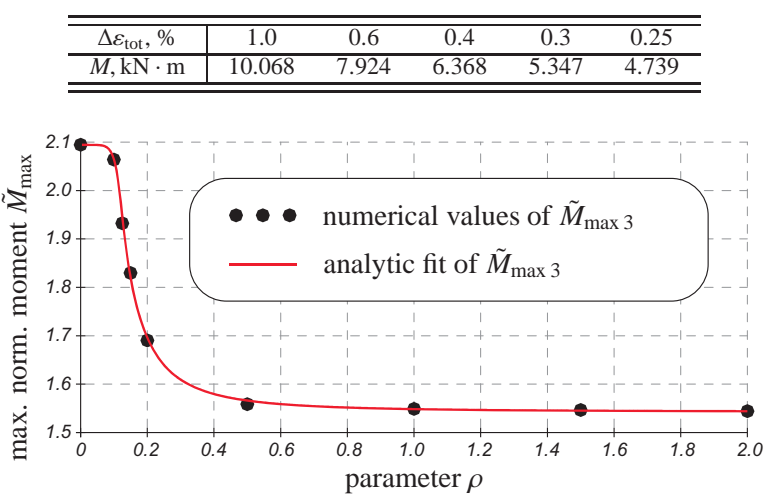

Figure 4: Numerical values of maximum normalised moment $\tilde{M}_{\max }$ from Table 3 fitted by Eq. (18)

\subsection{Evaluation of limit load}

It is usually preferable to convert the absolute values of bending moment $M$ into values of normalised bending moment $\tilde{M}$, which is suitable for the formulation of an analytic assessment model for number of cycles to creepfatigue failure $N^{\star}$, as proposed in [6]. Referring to [6], $\tilde{M}$ is defined as the relation of variable bending moment range $\Delta M$ to shakedown limit $\Delta M_{\mathrm{sh}}$ :

$$
\tilde{M}=\Delta M / \Delta M_{\text {sh }},
$$

where $M_{\mathrm{sh}}$ is called initial yielding moment [30] and corresponds to the loading conditions, when yielding is just beginning at the edge of a beam profile.

The limit load and shakedown limit are evaluated with an elastic-perfectly-plastic (EPP) model and a von Mises yield condition using material properties corresponding to the saturated cyclic plasticity response $\left(E, \sigma_{\mathrm{y}}\right.$ and $\left.v\right)$ reported in Table 1 of [6] for the steel AISI type $316 \mathrm{~N}(\mathrm{~L})$ at $550^{\circ} \mathrm{C}$. 
Table 3: The values of maximum normalised bending moment $\tilde{M}_{\max }$ obtained numerically for type 3 weldment

\begin{tabular}{c|ccccc|cc|cc}
\hline \hline Conf. & 1 & 2 & 3 & 4 & 5 & 8 & 9 & 6 & 7 \\
\hline$\rho$ & 2.0 & 1.5 & 1.0 & 0.5 & 0.2 & 0.15 & 0.125 & 0.1 & 0 \\
$\tilde{M}_{\max }$ & 1.54405 & 1.54644 & 1.54884 & 1.5585 & 1.69036 & 1.82967 & 1.9323 & 2.06405 & 2.09434 \\
\hline \hline
\end{tabular}

In the case of a rectangular cross-section plate in bending, assuming plane strain conditions (9), $M_{\text {sh }}$ is defined analytically according to [30] as

$$
M_{\mathrm{sh}}=\frac{\sigma_{\text {eop y } w h k^{2}}}{6} \text { with } \quad \sigma_{\text {eop y }}=\frac{2}{\sqrt{3}} \sigma_{\mathrm{y}} .
$$

The values of bending moment exceeding $M_{\mathrm{sh}}$ with further growth of plastic strain gradually approach the limit load value or fully plastic moment, which is defined analytically [30] as

$$
M_{\text {lim }}=\sigma_{\text {eop y }} w t h k^{2} / 4 \text {. }
$$

When $M$ reaches the value of $M_{\text {lim }}$, it is assumed that the plate cross-section is completely in plastic flow leading to a plastic hinge and structural collapse. It should be noted that the ratio $M_{\mathrm{lim}} / M_{\mathrm{sh}}=1.5$ changes if the cross-sectional shape is not rectangular or if a plate with rectangular cross-section contains welds. Refer to [30] for other cases of a beam cross-section. In particular case of types 1,2 or 3 weldments availability, the value of $M_{\text {lim }}$ remains the same, because the $\sigma_{\mathrm{y}}$ values of weld associated materials are usually higher than the $\sigma_{\mathrm{y}}$ of parent material. So plastic hinge usually occurs in locations remote from weld for uniformly distributed bending moment. At least, this assumption is true for the steel AISI type $316 \mathrm{~N}(\mathrm{~L})$ at $550^{\circ} \mathrm{C}$ [6]. However, the value $M_{\mathrm{sh}}$ for welded plate usually decreases, since the yielding starts at lower values of applied bending moment $M$ comparing to whole plate, because of material and geometry non-uniformity. In [6], this ratio was called the maximum normalised bending moment

$$
\tilde{M}_{\max }=\Delta M_{\text {lim }} / \Delta M_{\text {sh }} \text {. }
$$

Parameter $\tilde{M}_{\max }$ had a value of 1.551 for type 2 dressed weldment as defined in [6], and values in ranges from 1.509 for dressed to 2.323 for as-welded type 1 weldments and from 1.516 for dressed to 2.301 for as-welded type 2 weldments. So $\tilde{M}_{\max }$ is dependent on the particular geometric configuration of the weldment, and therefore should be taken into account in the formulation of parametric relations. Following this assumption and Eqs (13) and (16) the normalised bending moment is introduced in the following form:

$$
\tilde{M}=\frac{M}{M_{\mathrm{sh}}}=\frac{M \tilde{M}_{\max }}{M_{\mathrm{lim}}} \text { with } \quad M_{\mathrm{lim}}=\frac{\sigma_{\mathrm{y}} w t h k^{2}}{2 \sqrt{3}} .
$$

Thus, the awareness of the parent material yield stress $\sigma_{\mathrm{y}}$ of the steel AISI type $316 \mathrm{~N}(\mathrm{~L})$ reported in Table 1 of [6] and geometrical parameters of specimen $(t h k=26 \mathrm{~mm}$ and $w=200 \mathrm{~mm}$ ) allows the calculation of the limit bending moment as $M_{\mathrm{lim}}=10.564[\mathrm{kN} \cdot \mathrm{m}]$ for the conditions of experiments [8, 9, 10, 11]. If the weld geometry is the same as in the cruciform weldment specimens, then $\tilde{M}_{\max }=1.551$ and the values of normalised bending moment $\tilde{M}$ in experiments $[8,9,10,11]$ are calculated as reported in Table 4 of [6]. For other geometrical configurations of weldments, the set of $\tilde{M}$ will be slightly different, because $\tilde{M}_{\max }$ is individual for each weld type and geometrical configuration and were estimated numerically using step-by-step FEA.

Table 3 lists not only the values of $\tilde{M}_{\max }$ corresponding to the configurations defined in Table 1 for type 3 weldments, but it also contains values of $\tilde{M}_{\max }$ for two additional configurations 8 and 9 defined by $\rho$ of 0.15 and 0.125 . These all values are calculated by Eq. (16), which includes the values of $M_{\mathrm{lim}}$ and $M_{\mathrm{sh}}$ obtained numerically for each of the 9 configurations using step-by-step FEA with an EPP material model. Using the values of $M$ from Table 2, the values of $\tilde{M}_{\max }$ reported in Table 3 and the value of $M_{\mathrm{lim}}=10.564[\mathrm{kN} \cdot \mathrm{m}]$, the values of normalised moment $\tilde{M}$ for each configuration and each $\Delta \varepsilon_{\text {tot }}$ can be calculated by Eq. (17). Thus, in order to provide the values of $\tilde{M}$ in fully analytical form, the values of $\tilde{M}_{\text {max }}$ are defined as dependent on the geometric parameter of the weld profile $\rho$ :

$$
\begin{aligned}
& \tilde{M}_{\max 3}(\rho)=f_{1}(\rho)[1-H(\rho)]+f_{2}(\rho) H(\rho) \text { with } \\
& f_{1}(\rho)=m_{1}+m_{2} \rho^{m_{3}}, \quad f_{2}(\rho)=m_{4}+m_{5} \rho^{m_{6}} \text { and } H(\rho)=0.5+0.5 \tanh \left(\frac{\rho-m_{7}}{m_{8}}\right) .
\end{aligned}
$$




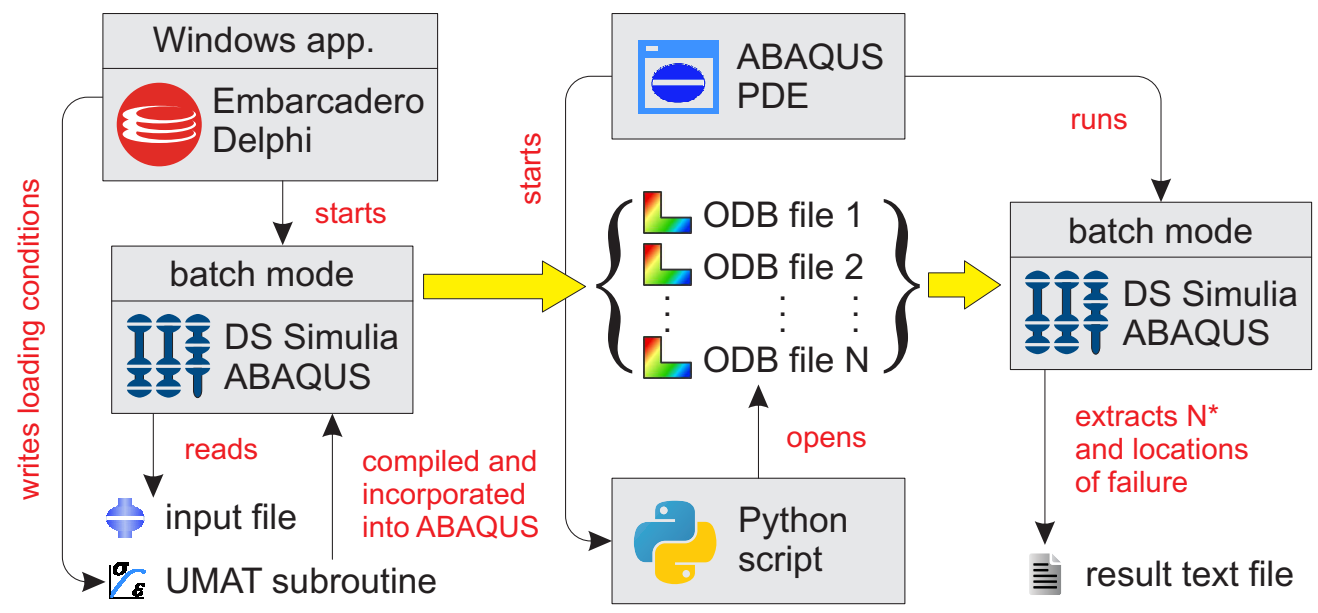

Figure 5: Flowchart of the parametric study implementation using several analysis automation techniques

In notation (18) constants $m_{1}=2.09434, m_{2}=-9.92412 \cdot 10^{5}$ and $m_{3}=7.51539$ are fitting parameters of the first power-law part $f_{1}(\rho)$, and constants $m_{4}=1.5427, m_{5}=5.59388 \cdot 10^{-3}$ and $m_{6}=-5.59388$ are fitting parameters of the second power-law part $f_{2}(\rho)$. Constant $m_{7}=0.12$ is the value of $\rho$ corresponding to intersection of functions $f_{1}(\rho)$ and $f_{2}(\rho)$, and $m_{8}=0.001$ is the smoothing parameter in an analytic approximation $H(\rho)$ of the Heaviside step function. The result of fitting the $\tilde{M}_{\max 3}$ numerical values from Table 3 by the analytic function $\tilde{M}_{\max 3}(\rho)$ in the form of Eq. (18) is illustrated in Fig. 4.

Finally, taking Eq. (15) for the bending moment $M$ and Eq. (8) for the fully plastic moment $M_{\text {lim }}$, which are both dependent on material properties $\left(E, v, B, \beta, \sigma_{\mathrm{y}}\right)$ and parameters of plate cross-section ( $w$ and $\left.t h k\right)$, and Eq. (18) for the maximum normalised moment $\tilde{M}_{\max 3}$ dependent on parameter of weld profile $\rho$, and using them in Eq. (17) results in the fully parametric formulation of the normalised bending moment dependent on total strain $\operatorname{range} \tilde{M}\left(\Delta \varepsilon_{\text {tot }}\right)$.

\section{Structural integrity assessments}

\subsection{Numerical creep-fatigue evaluation}

Since the objective of the research is a formulation of parametric relations able to describe long-term structural integrity of weldments, the creep-fatigue strength of each configuration from Table 1 should be evaluated in a wide range of loading conditions. These conditions are presented by different combinations of $\Delta \varepsilon_{\text {tot }}$ in the parent plate outer fibre, as a characteristic of fatigue effects, and duration $\Delta t$ of dwell period, as a characteristic of creep effects. The set of 5 values for $\Delta \varepsilon_{\text {tot }}$ is the same as in the experimental studies $[8,9,10,11]$, see Table 2 . The set of $\Delta t$ values used is the same as in the previous simulation study [6]: 0, 0.5, 1, 2, 5, 10, 100, 1000 and 10000 hours. Therefore, for each of 7 configurations for type 3 weldment 45 creep-fatigue evaluations must be performed with different values of $\Delta \varepsilon_{\text {tot }}$ and $\Delta t$, analogically to [25] for type 1 and 2 weldments. In the scope of parametric study, 315 FE-simulations of the parametric models shown in Fig. 2 have been carried out, using the LMM method, material models and constants given in [6]. The outputs of the LMM have been processed by the creep-fatigue procedure proposed in [6] to evaluate all the values of number of cycles to failure evaluate $N^{\star}$. The concept of the proposed creep-fatigue evaluation procedure, considering time fraction rule for creep-damage assessment, is explained in detail in [6] and consists of 5 steps including estimation of:

1. Saturated hysteresis loop using the LMM;

2. Fatigue damage using S-N diagrams;

3. Stress relaxation with elastic follow-up;

4. Creep damage using creep rupture curves;

5. Total damage using an interaction diagram. 

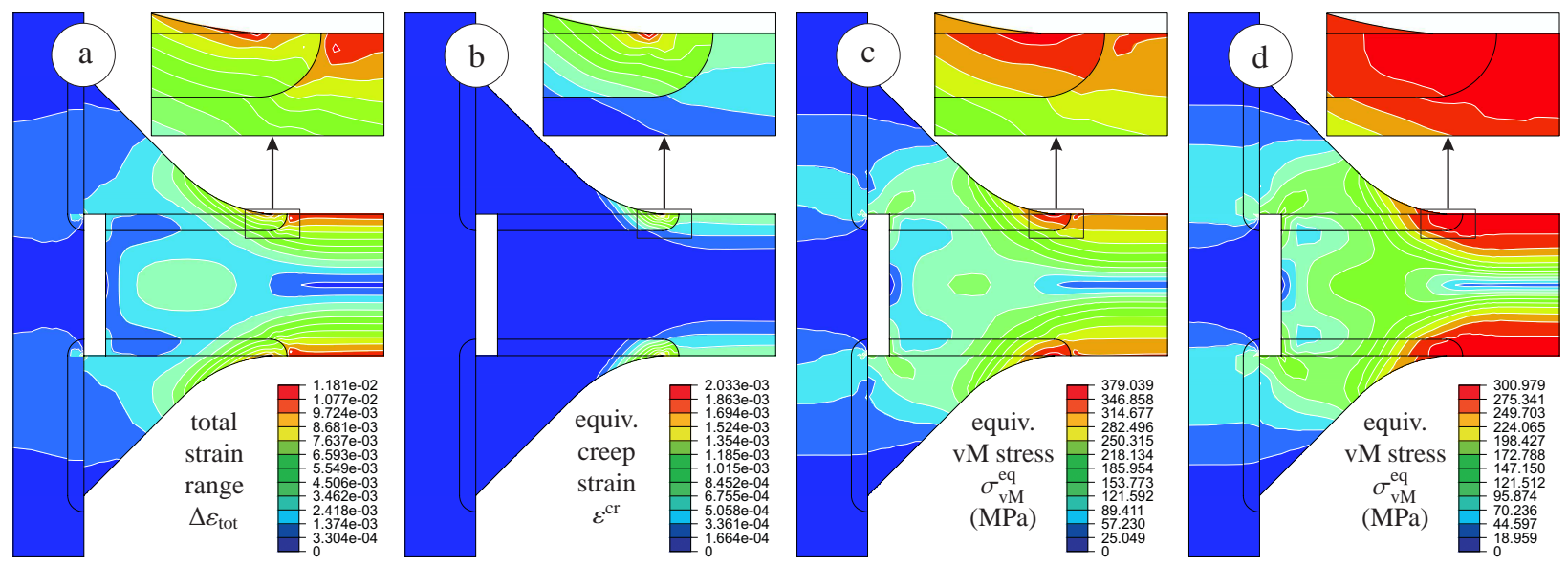

Figure 6: Contour plots of LMM results for type 3 weldment corresponding to $\Delta \varepsilon_{\text {tot }}=1 \%$ on the outer fiber of plate and $\Delta t=5$ hours of dwell: a) total strain range $\Delta \varepsilon_{\text {tot }}$; b) equivalent creep strain $\varepsilon^{\mathrm{cr}} ; \mathrm{c}$ ) equivalent von Mises stress $\varepsilon_{\mathrm{vM}}^{\mathrm{eq}}$ at the beginning of dwell; d) equivalent von Mises stress $\varepsilon_{\mathrm{vM}}^{\mathrm{eq}}$ at the end of dwell

Since the LMM requires lower computational effort compared to other methods, it appears to be an effective tool for express analysis of a large number of different loading cases using automation techniques. In order to carry out hundreds of FE-simulations in CAE-system ABAQUS and retrieve corresponding values of $N^{\star}, 3$ analysis improvements using automation techniques have been developed in this parametric study, which are illustrated in the form of flowchart in Fig. 5. The key elements of this flowchart are following:

1. The stand-along application allows running automatically the sequence of analyses with different values of applied bending moment and dwell period.

2. The UMAT subroutine written in FORTRAN comprises all 5 steps of the proposed creep-fatigue evaluation procedure, and implementation of the LMM and material models.

3. In order to process the results from hundreds of ODB-files, a Python script has been developed, which identifies the locations with minimum number of cycles to failure and extracts them into text file along with numbers of element and node.

The first automation technique is the development of a stand-alone application using Embarcadero Delphi integrated development environment using Delphi programming language. This simple application automatically carries out the sequence of all FE-simulations with different values of $M$ and $\Delta t$ for each configuration from Table 1 . This is implemented by automated modification of the UMAT subroutine including changing of loading values $(M$ and $\Delta t)$ and output file names, therefore producing individual ABAQUS result ODB-file for each loading case.

The second automation technique is the embedding of all 5 steps of the proposed creep-fatigue evaluation procedure in FORTRAN code of user material subroutine UMAT containing the implementation of the LMM and material models described in [6]. For a detailed description of the numerical procedure for the creep strain and flow stress estimation in the LMM code refer to [7, 24], and for a general guide to the LMM implementation using the ABAQUS user subroutines refer to [23]. The creep-fatigue evaluation procedure is implemented once the LMM has converged upon the stabilised cyclic behaviour. The LMM analysis was performed using three load instances in the cycle with creep dwell: 1) end of direct loading, 2) end of dwell period, 3) end of reverse loading. This results in a saturated hysteresis loop in terms of effective strain and effective von Mises stress for each integration point in the FE-model, as shown in Fig. 5 of [6]. The most important parameters (derived in the 1st step of the procedure) for further creepfatigue evaluation are the total strain range $\Delta \varepsilon_{\text {tot }}$, stress $\sigma_{1}$ at the beginning of dwell period and the elastic follow-up factor $Z$. These parameters from each integration point with material properties for elasticity, fatigue and creep, defined in the ABAQUS input file, are transferred into a new subroutine. This subroutine implements the next 4 steps of the procedure [6], which calculates and outputs the following parameters into ABAQUS result ODB-file: time to creep rupture $t^{*}$, creep damage accumulated per cycle $\omega_{1 \mathrm{c}}^{\mathrm{cr}}$, number of cycles to fatigue failure $N^{*}$, fatigue damage accumulated per 1 cycle $\omega_{1 \mathrm{c}}^{\mathrm{f}}$, and the most important - total number of cycles to failure in creep-fatigue conditions 

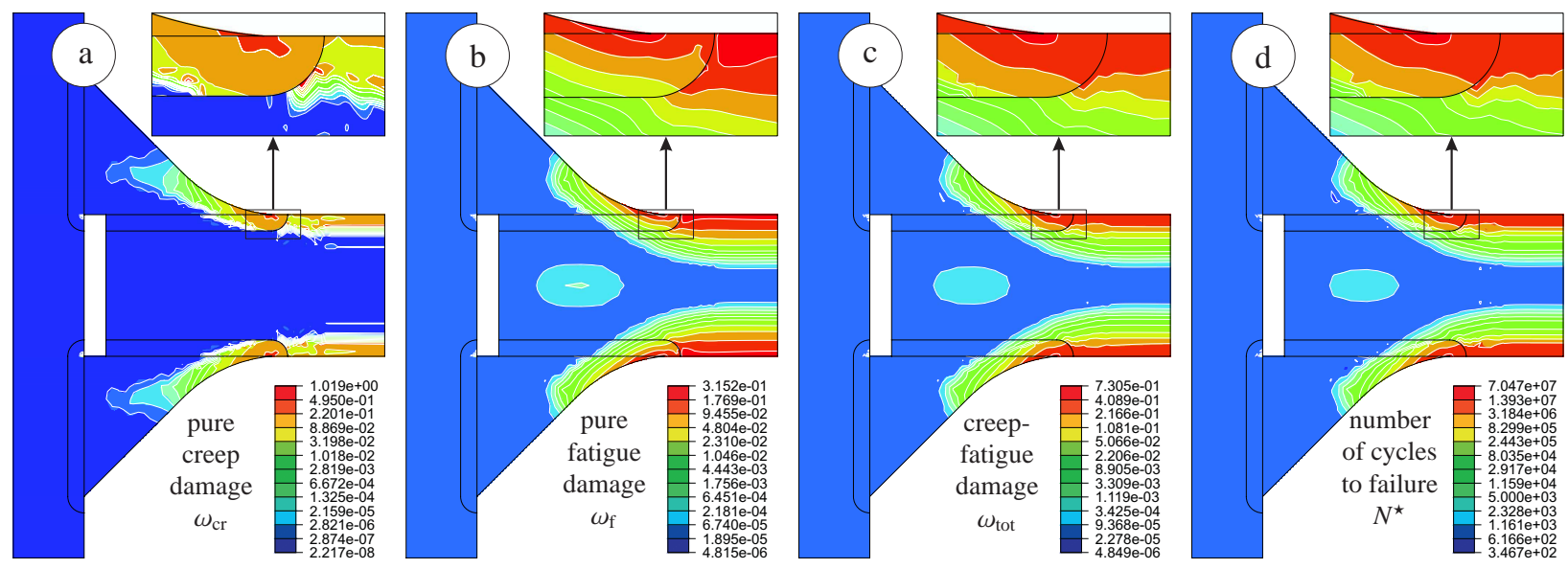

Figure 7: Contour plots of LMM results for type 3 weldment corresponding to $\Delta \varepsilon_{\text {tot }}=1 \%$ on the outer fiber and $\Delta t=5$ hours of dwell, which lead to creep-fatigue failure in weld toe after 379 cycles: a) pure creep damage $\omega_{\mathrm{cr}}$; b) pure fatigue damage $\omega_{\mathrm{f}}$; c) creep-fatigue damage $\omega_{\text {tot }}$; d) number of cycles to failure $N^{\star}$

$N^{\star}$ obtained using the damage interaction diagram proposed by Skelton and Gandy [29]. It should be noted that this evaluation procedure was implemented in previous work [6] using Excel spreadsheets only for the most critical locations, identified manually as sites of $\Delta \varepsilon_{\text {tot }}$ and $\sigma_{1}$ maximum values.

Examples of the FE results for the types 1 and 2 weldments in typically dressed (no. 2) configuration can be found in $[6,25]$. Those contour plots correspond to the loading case of $\Delta \varepsilon_{\text {tot }}=1 \%$ and $\Delta t=5$ hours. Figure 6 in [25] and Fig. 9 in [6] show the outputs of FEA with the LMM (obtained in Step 1) for type 1 and type 2 weldments correspondingly including $\Delta \varepsilon_{\mathrm{tot}}, \varepsilon^{\mathrm{cr}}, \varepsilon_{\mathrm{vM}}^{\mathrm{eq}}$ at the beginning of dwell and $\varepsilon_{\mathrm{vM}}^{\mathrm{eq}}$ at the end of dwell. Figure 7 and Fig. 5 in [25] show the outputs of the creep-fatigue evaluation procedure for type 1 and type 2 weldments correspondingly including $\omega_{\mathrm{cr}}, \omega_{\mathrm{f}}, \omega_{\text {tot }}$ and $N^{\star}$. The critical location with $N^{\star}=206$ for type 1 is the same as for analogical type 2 weldment with $N^{\star}=279$ - the corner element in the weld toe adjacent to HAZ.

Exactly the same approach is used to demonstrate an example of the type 3 weldment comprising the typically dressed configuration (no. 3 from Table 1) and loading case of $\Delta \varepsilon_{\text {tot }}=1 \%$ and $\Delta t=5$ hours. Figure 6 shows the outputs of FEA with the LMM, while Fig. 7 shows the outputs of the creep-fatigue evaluation procedure. The critical location with $N^{\star}=379$ for this type 3 is the same as for analogical types 1 and 2 weldments - the corner element in the weld toe adjacent to HAZ.

The distribution of total strain range $\Delta \varepsilon_{\mathrm{tot}}$, with maximum value $\Delta \varepsilon_{\mathrm{tot}}^{\max }=1.143 \%$ at the critical location, is shown in Fig. 6a. The distribution of equivalent creep strain $\varepsilon^{\mathrm{cr}}$ at load instance 2 with maximum value $\varepsilon_{\max }^{\mathrm{cr}}=1.745 \cdot 10^{-3}$ at the critical location is shown in Fig. 6b. The distribution of equivalent von Mises stress $\sigma_{\mathrm{vM}}^{\mathrm{eq}}$ at the beginning of dwell at load instance 1 with value $\sigma_{1}^{\text {eq }}=320.712 \mathrm{MPa}$ at the critical location is shown in Fig. 6c. The distribution of equivalent von Mises stress $\sigma_{\mathrm{vM}}^{\mathrm{eq}}$ at the end of dwell at load instance 2 with value $\sigma_{2}^{\mathrm{eq}}=276.063 \mathrm{MPa}$ at the critical location is shown in Fig. 6d. Therefore, the drop of stress $\Delta \sigma^{\mathrm{eq}}=44.649 \mathrm{MPa}$ during $\Delta t=5$ hours of dwell provides the value of elastic follow up factor $Z=5.5$.

The distribution of pure creep damage $\omega_{\mathrm{cr}}$ with maximum value $\omega_{\mathrm{cr}}^{\max }=1.001 \cdot 10^{-3}$ at the critical location is shown in Fig. 7a. The distribution of pure fatigue damage $\omega_{\mathrm{f}}$ with maximum value $\omega_{\mathrm{f}}^{\max }=0.763 \cdot 10^{-3}$ at the critical location is shown in Fig. 7b. The distribution of total damage $\omega_{\text {tot }}$ with maximum value $\omega_{\text {tot }}^{\max }=1.765 \cdot 10^{-3}$ at the critical location is shown in Fig. 7c. The distribution of $N^{\star}$ with minimum value $N_{\mathrm{min}}^{\star}=379$ at the critical location is shown in Fig. 7d.

In spite of the same critical location and almost equal values of the accumulated total damage at failure for types 1 , 2 and 3 weldments, type 1 has minimum residual life compared to other types, because of higher values of parameters characterising the hysteresis loop $\left(\Delta \varepsilon_{\mathrm{tot}}, \varepsilon^{\mathrm{cr}}, \sigma_{1}^{\mathrm{eq}}, \sigma_{2}^{\mathrm{eq}}\right.$ and $\left.Z\right)$. The reduced residual life is effected by the smaller weld deformation stiffness caused by smaller overall size of the type 1 weld profile compared to types 2 and 3 .

The third automation technique is the development of a script using ABAQUS Python Development Environment (Abaqus PDE) using Python programming language [31]. This script, when started in ABAQUS environment, appends 


\begin{tabular}{c|lllllll}
\hline \hline Conf. & No. 1 & No. 2 & No. 3 & No. 4 & No. 5 & No. 6 & No. 7 \\
\hline$\rho$ & 2.0 & 1.5 & 1.0 & 0.5 & 0.2 & 0.1 & 0 \\
\hline$a_{0}$ & 0.241276 & 0.244349 & 0.246553 & 0.259228 & 0.281809 & 0.279272 & 0.289034 \\
$a_{1}$ & 0.081673 & 0.084144 & 0.084864 & 0.078608 & 0.053713 & 0.044155 & 0.040990 \\
$a_{2}$ & -0.034886 & -0.036391 & -0.036562 & -0.032019 & -0.015003 & -0.005038 & -0.001699 \\
$a_{3}$ & 0.009661 & 0.009863 & 0.009823 & 0.008879 & 0.004838 & 0.001026 & 0.000302 \\
$b_{0}$ & 0.699869 & 0.687317 & 0.675843 & 0.581696 & 0.537877 & 0.586562 & 0.623805 \\
$b_{1}$ & 0.269679 & 0.251640 & 0.243094 & 0.348330 & 0.271017 & 0.137853 & 0.137519 \\
$b_{2}$ & -0.214709 & -0.200898 & -0.198880 & -0.259515 & -0.211524 & -0.110712 & -0.111733 \\
$b_{3}$ & 0.052596 & 0.050037 & 0.050015 & 0.059313 & 0.051960 & 0.033343 & 0.032513 \\
\hline \hline
\end{tabular}

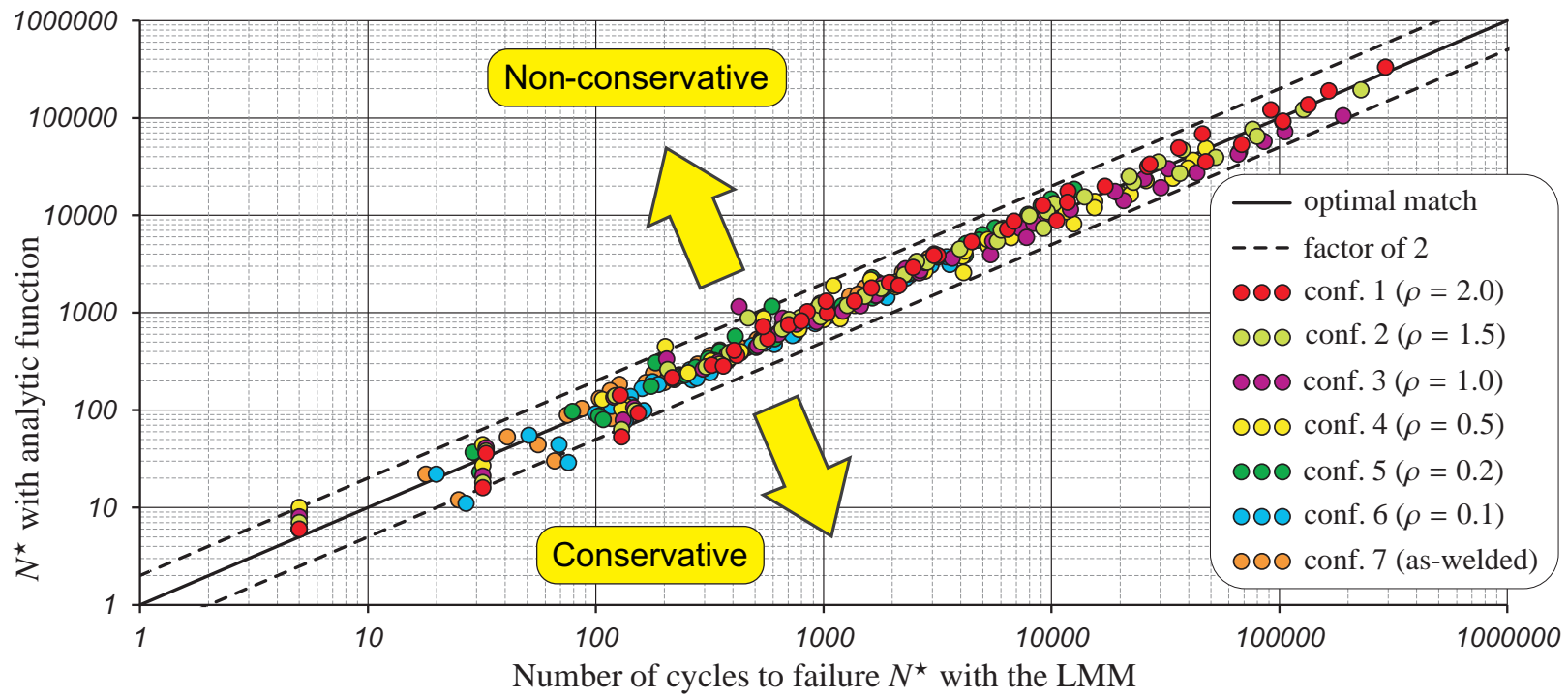

Figure 8: Comparison of $N^{\star}$ obtained with the LMM and the analytic function (19) for type 3 weldment

the list of available result ODB-files corresponding to one configuration. For each of ODB-files, it reads the values of $N^{\star}$ in each integration point, selects the integration point with minimum value of $N^{\star}$ over the FE-model, and writes the numbers of element and integration point and material name to an output text file. Therefore, the critical locations and corresponding values of $N^{\star}$ are extracted automatically for all configurations and loading cases. Obtained results can be used for the formulation of an analytic assessment model suitable for the fast estimation of $N^{\star}$ for a variety of loading conditions $(\tilde{M}$ and $\Delta t)$ and geometrical parameter $\rho$ values.

\subsection{Analytic assessment model}

For each of the 7 configurations from Table 1, the array of assessment results consisting of 45 values of $N^{\star}$ corresponding to particular values of $\tilde{M}$ and $\Delta t$ is fitted using the least squares method by the following function proposed in the form of power-law in $[6,25]$ :

$$
\log \left(N^{\star}\right)=\tilde{M}^{-b(\Delta t)} / a(\Delta t)
$$

where the fitting parameters dependent on $\Delta t$ are

$$
\begin{aligned}
& a(\Delta t)=a_{3} \log (\Delta t+1)^{3}+a_{2} \log (\Delta t+1)^{2}+a_{1} \log (\Delta t+1)+a_{0} \quad \text { and } \\
& b(\Delta t)=b_{3} \log (\Delta t+1)^{3}+b_{2} \log (\Delta t+1)^{2}+b_{1} \log (\Delta t+1)+b_{0},
\end{aligned}
$$


and the independent fitting parameters $\left(a_{0}-a_{3}\right.$ and $\left.b_{0}-b_{3}\right)$ have particular values, which are individual for each type of weldment and each available configuration. For type 3 weldment they are reported in in Table (4).

In order to capture all configurations with an unified set of fitting parameters, parameters $a_{0}-a_{3}$ and $b_{0}-b_{3}$ from Table 4 should be defined as dependent on geometric parameter $\rho$ using the least squares method:

$$
\begin{array}{ll}
a_{0}^{\mathrm{T} 3}(\rho)=-4.506 \cdot 10^{-2} \ln (\rho+1)+0.285, & b_{0}^{\mathrm{T} 3}(\rho)=0.118 \ln (\rho+1)+0.57, \\
a_{1}^{\mathrm{T} 3}(\rho)=4.1 \cdot 10^{-2} \ln (\rho+1)+4.701 \cdot 10^{-2}, & b_{1}^{\mathrm{T} 3}(\rho)=8.742 \cdot 10^{-2} \ln (\rho+1)+0.195, \\
a_{2}^{\mathrm{T} 3}(\rho)=-3.202 \cdot 10^{-2} \ln (\rho+1)-7.575 \cdot 10^{-3}, & b_{2}^{\mathrm{T} 3}(\rho)=-7.197 \cdot 10^{-2} \ln (\rho+1)-0.152, \\
a_{3}^{\mathrm{T} 3}(\rho)=8.74 \cdot 10^{-3} \ln (\rho+1)+2.10773 \cdot 10^{-3}, & b_{3}^{\mathrm{T} 3}(\rho)=1.397 \cdot 10^{-2} \ln (\rho+1)+4.034 \cdot 10^{-2} .
\end{array}
$$

The verification of the fit quality using the the geometrical parameter $\rho$ for the proposed relations (21) is implemented by applying Eqs (19) and (20) to estimate $N^{\star}$. Number of cycles to failure $N^{\star}$ is estimated for each of the 7 configurations using the corresponding values of ratio from Table 1 and for the same load combinations as were used for the LMM analyses. The results of the verification are illustrated on diagram in Fig. 8 for type 3 weldments in the form of $N^{\star}$ obtained with the analytic function (19) vs. $N^{\star}$ obtained with the LMM. Comparison of the analytic and numeric $N^{\star}$ shows that the quality of analytic predictions is quite close to the line of optimal match and provides a uniform scatter of results through all variants of loading conditions and configurations. The discrepancy between analytic predictions and numerical LMM outputs is generally within the boundaries of an inaccuracy factor equal to 2, which is allowable for engineering analysis, producing both conservative and non-conservative results.

It should be noted that the scatter of $N^{\star}$ for type 3 weldments approximately belongs to the range from 5 to 300,000 as shown in Fig. 8. It is slightly different from the scatter for type 2 weldments (approx. from 4 to 100,000) shown in Fig. 9 of [25] and the scatter for type 1 weldments (approx. from 2 to 500,000) shown in Fig. 8 of [25]. This observation shows that type 1 weldment is less creep-fatigue resistant than types 2 and 3 weldments in the same ranges of loading conditions and manufacturing variations. This fact could be explained by the significantly smaller amount of weld and parent material used for manufacturing of type 1 weldment compared to the types 2 and 3 for the same plate thickness, resulting in less rigidity and load-bearing capacity for type 1 weldment. Another important observation is that the average creep-fatigue resistivity of configuration no. 1 (perfectly dressed) is relatively the highest among all configurations. The average resistivity is slightly reducing from one configuration to another with the reduction of ratio $\rho$ value as shown in Fig. 8, resulting in the minimum average $N^{\star}$ for the configuration no. 7 (as-welded).

Having defined the number of cycles to failure $N^{\star}$ by Eq. (19), the residual service life in years is dependent on the duration of 1 cycle, which consists of dwell period $\Delta t$ and relatively short time of deformation:

$$
L^{\star}=N^{\star}\left[\frac{\Delta t}{365 \cdot 24}+\frac{2 \Delta \varepsilon_{\mathrm{tot}}(\tilde{M})}{\dot{\varepsilon}(365 \cdot 24 \cdot 60 \cdot 60)}\right],
$$

where $\dot{\varepsilon}=0.03 \% / \mathrm{s}$ is a strain rate according to experimental conditions [8, 9, 10, 11], and the parametric analytical relations for $\Delta \varepsilon_{\mathrm{tot}}(\tilde{M})$ are derived in Sect. 3. These relations consist of Eqs (1), (11) and (12) given in Sect. 3.1 to evaluate $\Delta \varepsilon_{\text {tot }}(\Delta \sigma(M))$, where $M$ is replaced by $\tilde{M}$ and $M_{\text {lim }}$ using Eq. (17) and $\tilde{M}_{\text {max }}$ using Eq. (18) given in Sect. 3.2 . The aforementioned group of equations for the relation $\Delta \varepsilon_{\text {tot }}(\tilde{M})$ include the geometrical parameters of parent plate cross-section (thk and $w$ ) and weld profile $(\rho)$, and parent plate material parameters $\left(E, v, B, \beta, \sigma_{\mathrm{y}}\right)$. This group of equations (1), (11), (12), (17) and (18) replaces Eq. (34) from [6], which is suitable for only one particular variant of weldment (type 2), weld profile (conf. 2 - typically dressed) and parent plate cross-section [8, 9, 10, 11].

\section{Parametric formulation of FSRF}

Since fatigue experimental data is usually obtained from an ideal controlled test environment, a Fatigue Strength Reduction Factor (FSRF) is an usual approach, which allows to account for a real working environment resulting in a reduction of residual life. For example in [32], a FSRF is introduced as scaling factor in stress-life approach to account for the influence of aggressive corrosive environment. The $\mathrm{S}-\mathrm{N}$ curve obtained in vacuum is used as a basic curve, while the curves for different grades of corrosive effect can be evaluated by transformation of the basic S-N curve using a corresponding FSRF. The same approach can be extended to strain-life approach, where FSRF is calculated as 
a relation between the strain range on the basic fatigue curve and the strain range on the effected fatigue curve leading to the same number of cycles to failure, and it is also usually averaged over the defined strain range. In strain-life approach FSRF accounts for influence of temperature increase or creep dwell period increase or geometrical effects such as introduction of weld or change of weld profile. In this research, the basic curve is the fatigue curve obtained for whole plate manufactured of the parent material - steel type $316 \mathrm{~N}(\mathrm{~L})$ at $550^{\circ} \mathrm{C}$ without creep dwell periods. Effected curves are the curves for welded plates obtained with different dwell periods and for different weld profile geometries, as shown in Fig. 9.

Since the function $N^{\star}(\tilde{M}, \Delta t)$ proved its validity in the previous subsection, it can be applied for the fast creepfatigue assessments of new welded structures during the design stage. However, it is generally hard to conclude about the service conditions $(\tilde{M}, \Delta t)$ required to estimate particular value of $N^{\star}$. Loading conditions comprise a wide range of mechanical loading described by $\tilde{M}$ or corresponding range of $\Delta \varepsilon_{\text {tot }}$ in parent material adjacent to welded joints. Thus, introduction of a FSRF allows a wide range of mechanical loading relevant to application area of a designed welded structure to be captured. The FSRF is usually determined experimentally $[8,9,10,11,33,34]$ by comparing the fatigue failure data of the welded specimen with the fatigue curve derived from tests on the parent plate material.

Table 5: FSRFs for pure fatigue of type 3 weldments

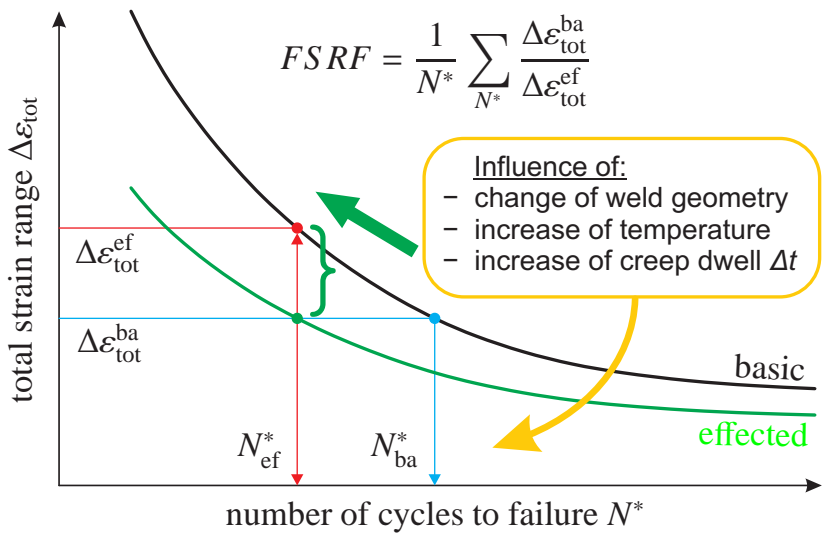

Figure 9: Purpose of FSRF for fatigue strain-life approach in application to creep-fatigue endurance of weldments

\begin{tabular}{l|ccccccc}
\hline \hline Conf. & 1 & 2 & 3 & 4 & 5 & 6 & 7 \\
\hline FSRF & 1.3 & 1.425 & 1.595 & 1.872 & 2.362 & 3.252 & 3.46 \\
\hline \hline
\end{tabular}

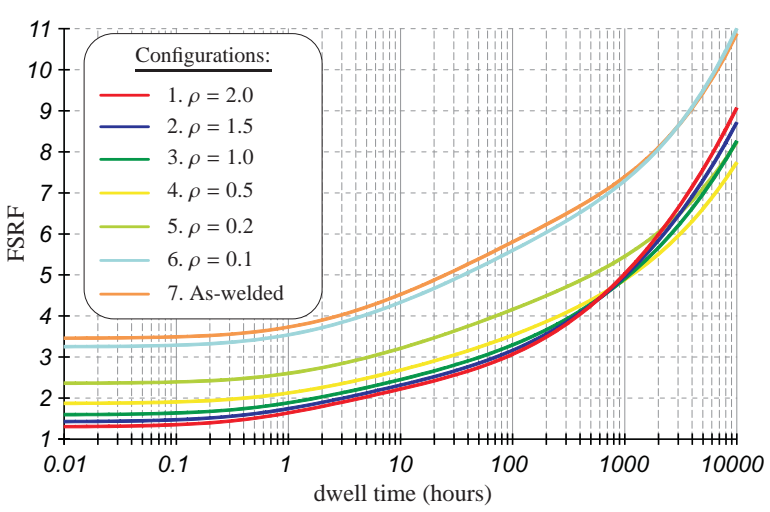

Figure 10: Dependence of FSRF on duration of $\Delta t$ for different configurations of type 3 weldments

The current approach in R5 Volume 2/3 Procedure [12] operates with the fixed values of FSRF for 3 different types of weldments taking into account dressed and as-welded variants, which consider only the reduction of fatigue strength of weldments compared to the parent material. For austenitic steel weldments [34, 33], FSRF = 1.5 is prescribed for both variants of type 1 , and FSRF $=1.5$ for type 2 dressed and FSRF $=2.5$ for as-welded variant, and FSRF $=3.2$ is prescribed for both variants of type 3. All this variety of the FSRFs is representative of the reduction in fatigue endurance caused by the local strain range $\varepsilon_{\text {tot }}$ enhancement in the weldment region due to the material discontinuity and geometric strain concentration effects. The introduction of FSRF as dependent on $\Delta t$ in [6] using function $N^{\star}(\tilde{M}, \Delta t)$ for the case of type 2 dressed weldment allowed the influence of creep to be taken into account, and to provide the adjusted values of FSRF for the real operation conditions, where creep-fatigue interaction takes place. Therefore, the same approach [6] is applied to obtain $\Delta t$-dependent FSRFs for a variety of geometrical configurations considering additional dependence on parameter of weld profile $\rho$.

For this purpose Eq. (19) is converted analytically to the relation $\tilde{M}\left(N^{\star}, \Delta t\right)$ and inserted into the group of relations $\Delta \varepsilon_{\mathrm{tot}}(\tilde{M})$ given in the end of previous subsection, resulting in the relation $\Delta \varepsilon_{\mathrm{tot}}\left(N^{\star}, \Delta t, \rho\right)$. This relation describes the $\Delta \varepsilon_{\text {tot }}$ in the parent material remote from weldment corresponding to particular values of $N^{\star}$ and $\Delta t$ for a particular geometrical configuration of weldment defined by $\rho$. Thus, the FSRFs, appropriate to varying values of $\Delta t$ and equal values of $N^{\star}$, are defined by the relation between the S-N diagram corresponding to fatigue failures of parent material 

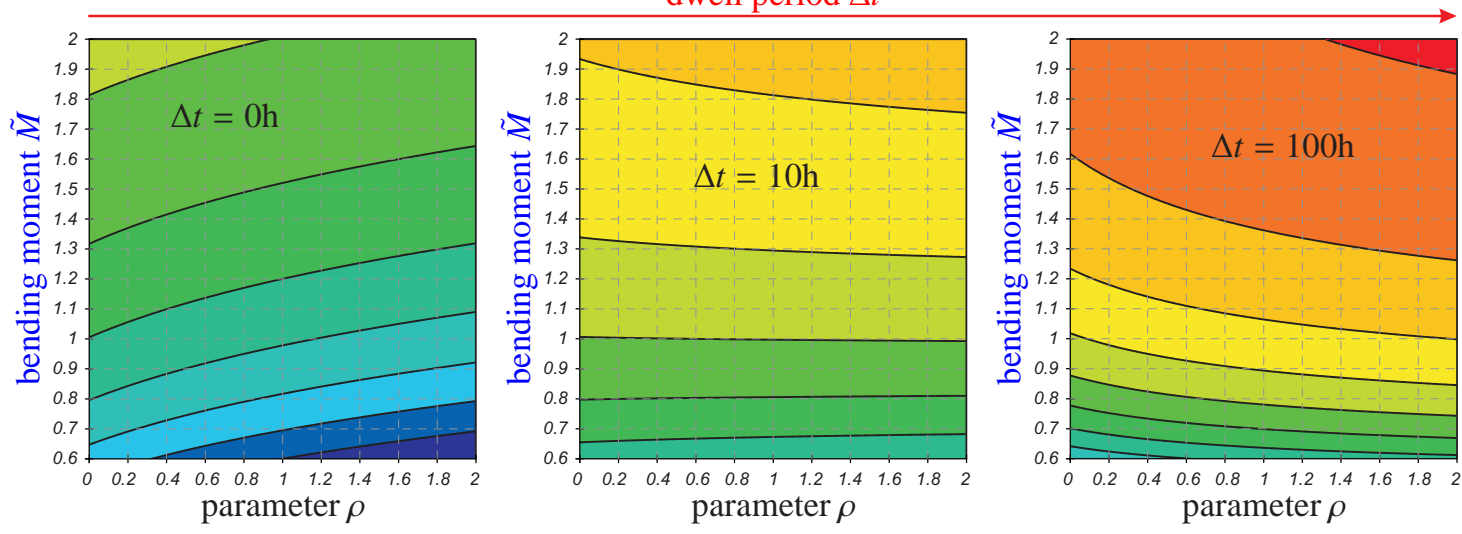

Figure 11: Contour plots for type 3 weldment characterising the influence of geometric parameter $\rho$ on number of cycles to failure $N^{\star}$ for different variant of dwell period $\Delta t$ obtained with Eqs (19) - (21)

plate and S-N diagrams for a weldment defined by $\rho$ :

$$
\mathrm{FSRF}=\Delta \varepsilon_{\text {tot }}^{\mathrm{par}}\left(N^{\star}\right) / \Delta \varepsilon_{\mathrm{tot}}\left(N^{\star}, \Delta t, \rho\right),
$$

where the S-N diagram for parent material plate is

$$
\log \left(\Delta \varepsilon_{\text {tot }}^{\mathrm{par}}\right)=p_{0}+p_{1} \log \left(N^{*}\right)+p_{2} \log \left(N^{*}\right)^{2},
$$

with the following polynomial coefficients referring to [33]: $p_{0}=2.2274, p_{1}=-0.94691$ and $p_{2}=0.085943$.

The FSRFs estimated by Eq. (23) corresponding to the range of $\Delta t \in\left[0 \ldots 10^{5}\right]$ hours are defined in some particular range of $N^{\star}$. This range is different for each value of $\Delta t$ characterised by reducing value of the average $N^{\star}$ with the growth of $\Delta t$. The upper bound of the $N^{\star}$ range is governed by the mathematical upper limit of the S-N diagram $\Delta \varepsilon_{\text {tot }}^{\text {par }}\left(N^{\star}\right)$ for parent material plate, which is defined in [6] as $\log \left(N_{\text {max }}^{\star}\right)=p_{1} /\left(2 p_{2}\right)=5.51$ or $\Delta \varepsilon_{\text {tot }}^{\text {par }}\left(10^{5.51}\right)=0.416 \%$. The lower bound of the $N^{\star}$ range is flexible and governed by $\Delta t$ using the following function:

$$
\log \left(N_{\min }^{\star}\right)=3-0.5 \log (\Delta t+1) .
$$

Finally, for each of the 7 configurations from Table 1 the FSRF is defined as a continuous function of $\Delta t$ using Eq. (23) using simple averaging procedure over a dynamic range of $N^{\star}$ from $\log \left(N_{\text {min }}^{\star}\right)$ to $\log \left(N_{\text {max }}^{\star}\right)$ with step 0.01 . The resultant dependencies of FSRFs on $\Delta t$ are illustrated in Fig. 10 for type 3 weldments with designation of different configurations. First of all, this figure shows significant enhancement of FSRF for dwells $\Delta t>0.1$ hour caused by creep, which is important for design applications. The initial values of FSRFs corresponding to pure fatigue conditions $(\Delta t=0)$ are listed in Table 5 and could be compared with the values recommended in R5 Volume 2/3 Procedure [12].

The FSRF for type 3 dressed weldments is within the range 1.302-1.425, for type 3 welded joints with moderate TIG dressing it is within the range 1.425-2.362 depending on the amount of TIG dressing, while R5 also doesn't give any value for these cases. The FSRF for type 3 as-welded joints without any additional treatment may reach up to 3.252-3.459, while R5 gives the value 3.2, which approximately corresponds to lower bound for the obtained range. It should be noted that the value of FSRF for type 3 recommended by R5 procedure may be significantly conservative, if some kind of TIG dressing is applied.

\section{Conclusions}

The parametric study on creep-fatigue strength of the steel AISI type 316N(L) weldments of type 3 according to classification of R5 Vol. 2/3 Procedure [12] at 550 ${ }^{\circ} \mathrm{C}$ has been implemented using the LMM. The study is based upon the latest developed creep-fatigue evaluation procedure [6] considering time fraction rule for creep-damage 
assessment. This procedure has been validated in [6] against experimental data $[8,9,10,11]$ comprising reverse bending tests of cruciform weldments for different combination of loading conditions.

Parametric model shown in Figs 1 and 2 allows the variation of parameter $\rho$ governing shape of the weld profile and loading conditions ( $\Delta t$ and $\tilde{M}$ ). Seven configurations, characterised by individual values of $\rho$ listed in Table 1 , are proposed to present different grades of TIG dressing. For each configuration, the total number of cycles to failure $N^{\star}$ in creep-fatigue conditions is assessed numerically for different loading cases using automation techniques shown in Fig. 5 and explained in Sect. 4.1. The obtained set of $N^{\star}$ is extrapolated by the analytic function (19) dependent on $\tilde{M}$ with fitting functions (20) dependent on $\Delta t$, which includes the fitting parameters (21) dependent on $\rho$.

Proposed function (19) for $N^{\star}$ shows good agreement with numerical results obtained by the LMM in Fig. 8. The discrepancy between analytic predictions and numerical LMM outputs is generally within the boundaries of an inaccuracy factor equal to 2, which is allowable for engineering analysis, producing both conservative and nonconservative results. Thus, it is used for the identification of FSRFs intended for design purposes and dependent on $\Delta t$ and $\rho$. The proposed function for FSRFs (23) is applied to all 7 configuration from Table 1 characterised by $\rho$ in order to obtain continuous dependencies on $\Delta t$, which are shown in Fig. 10. Therefore, this approach improves upon existing design techniques, e.g. in R5 Procedure [12], by considering the significant influence of creep. Moreover, the obtained FSRFs for pure fatigue revises the value recommended in R5 Procedure [12] removing the redundant conservatism for type 3 dressed weldments. In order to confirm the applicability of the proposed improvements, the analytical formulations for FSRFs still need final validation against some additional experiments.

Both numerical and analytical predictions (19) confirm that TIG-dressing can improve up to 10 times the residual life of type 3 weldments in pure fatigue conditions and up to $100 \%$ the residual life of weldments in creep-fatigue conditions. In order to conclude about the sensitivity of creep-fatigue strength to a variation of $\rho$, the Eqs (19) - (21) for $N^{\star}(\tilde{M}, \Delta t, \rho)$ are applied to create a set of contour plots shown in Fig. 11. These plots characterise the influence of geometrical parameter $\rho$ and mechanical loading intensity $\tilde{M}$ on $N^{\star}$ at 3 different variants of dwell period $\tilde{M}$ for type 3 weldment. The global tendency is that $\rho$ generally decreases the strength, while $\tilde{M}$ generally increases it. However the contribution of each parameter $(\rho$ and $\tilde{M})$ is dependent on type of creep-fatigue interaction characterised by the dwell duration $\Delta t$. The positive influence of $\rho$ is much more significant at pure fatigue conditions than under the creep-dominant conditions, where it is suppressed by stress relaxation caused by creep. Since the creep-relaxation reduces the stress in locations of its concentration, the intensity of mechanical loading becomes more important under pure creep conditions.

\section{Acknowledgements}

The authors deeply appreciate the Engineering and Physical Sciences Research Council (EPSRC) of the UK for the financial support in the frames of research grant no. EP/G038880/1, the University of Strathclyde for hosting during the course of this work, and EDF Energy for the experimental data.

\section{References}

1. Lee, Y.-L., Barkey, M.E., Kang, H.-T.. Metal Fatigue Analysis Handbook: Practical Problem-Solving Techniques for Computer-Aided Engineering. Oxford: Butterworth-Heinemann; 2012.

2. Macdonald, K.A.. Fracture and fatigue of welded joints and structures. Cambridge, UK: Woodhead Publishing Limited; 2011.

3. Łagoda, T.. Lifetime Estimation of Welded Joints. Berlin: Springer-Verlag; 2008.

4. Radaj, D., Sonsino, C.M., Fricke, W.. Fatigue Assessment of Welded Joints by Local Approaches. Cambridge: Woodhead Publishing Limited; 2nd ed.; 2006.

5. Guide to methods of assessing the acceptability of flaws in metallic structures. No. BS 7910:2013 in British Standard. London, UK: The British Standards Institution; 2013.

6. Gorash, Y., Chen, H.. Creep-fatigue life assessment of cruciform weldments using the linear matching method. Int J of Pressure Vessels $\mathcal{E}$ Piping 2013;104:1-13.

7. Chen, H.F., Chen, W., Ure, J.. A direct method on the evaluation of cyclic behaviour with creep effect. In: Proc. ASME Pressure Vessels $\mathcal{E}$ Piping Conf. (PVP2012); PVP2012-78065. Toronto, Canada: ASME; 2012, July 15-19.

8. Bretherton, I., Knowles, G., Slater, I.J., Yellowlees, S.F.. The fatigue and creep-fatigue behaviour of $26 \mathrm{~mm}$ thick type $316 \mathrm{~L}(\mathrm{~N})$ welded cruciform joints at $550^{\circ} \mathrm{C}$ : An interim report. Report for Nuclear Electric Ltd no. R/NE/432; AEA Technology plc; Warrington, UK; 1998.

9. Bretherton, I., Knowles, G., Bate, S.K.. PC/AGR/5087: The fatigue and creep-fatigue behaviour of welded cruciform joints: A second interim report. Report for BE Generation Ltd no. AEAT-3406; AEA Technology plc; Warrington, UK; 1999. 
10. Bretherton, I., Budden, P.J.. Assessment of creep-fatigue endurance of large cruciform weldments. In: Trans. 15th Int. Conf. on Structural Mechanics in Reactor Technology; SMiRT15 - F05/2. Seoul, Korea: IASMiRT; 1999, p. 185-192.

11. Bretherton, I., Knowles, G., Hayes, J.-P., Bate, S.K., Austin, C.J.. PC/AGR/5087: Final report on the fatigue and creep-fatigue behaviour of welded cruciform joints. Report for BE Generation Ltd no. RJCB/RD01186/R01; Serco Assurance; Warrington, UK; 2004.

12. Ainsworth, R.A., editor. R5: An Assessment Procedure for the High Temperature Response of Structures. Procedure R5: Issue 3. Gloucester, UK: British Energy Generation Ltd; 2003.

13. Nguyen-Tajan, T.M.L., Pommier, B., Maitournam, H., Houari, M., Verger, L., Du, Z.Z., et al. Determination of the stabilized response of a structure undergoing cyclic thermal-mechanical loads by a direct cyclic method. In: Proc. 16th Annual ABAQUS Users' Conference. Munich, Germany: Dassault Systèmes Simulia Corp.; 2003, June 4-6.

14. ABAQUS Analysis User's Manual. Dassault Systèmes Simulia Corp.; Version 6.10 ed.; 2010.

15. Ponter, A.R.S., Chen, H.F.. A minimum theorem for cyclic load in excess of shakedown, with application to the evaluation of a ratchet limit. European Journal of Mechanics - A/Solids 2001;20(4):539-553.

16. Chen, H.F., Ponter, A.R.S.. A method for the evaluation of a ratchet limit and the amplitude of plastic strain for bodies subjected to cyclic loading. European Journal of Mechanics - A/Solids 2001;20(4):555-571.

17. Birger, I.A.. Some general methods for solving problems of the theory of plasticity. J of Applied Mathematics and Mechanics 1951; 15(6):765-770.

18. Kachanov, L.M.. Fundamentals of the theory of plasticity. Amsterdam: North-Holland Publishing Company; 2 nd ed.; 1971.

19. Chen, H.F.. Lower and upper bound shakedown analysis of structures with temperature-dependent yield stress. J of Pressure Vessel Technology 2010;132(1):011202:1-8.

20. Chen, H.F., Ponter, A.R.S.. A direct method on the evaluation of ratchet limit. J of Pressure Vessel Technology 2010;132(4):041202:1-8.

21. Chen, H.F., Ponter, A.R.S.. Linear matching method on the evaluation of plastic and creep behaviours for bodies subjected to cyclic thermal and mechanical loading. Int J for Numerical Methods in Engineering 2006;68(1):13-32.

22. Ponter, A.R.S., Chen, H.F.. Modeling of the behavior of a welded joint subjected to reverse bending moment at high temperature. $J$ of Pressure Vessel Technology 2007;129(2):254-261.

23. Tipping, D.J.. The Linear Matching Method: A Guide to the ABAQUS User Subroutines. Generic Report no. E/REP/BBGB/0017/GEN/07; Central Engineering Support; BE Generation Ltd, Gloucester, UK; 2008.

24. Ure, J., Chen, H., Tipping, D.. Development and implementation of the ABAQUS subroutines and plug-in for routine structural integrity assessment using the Linear Matching Method. In: Proc. SIMULIA Regional User Meeting. Manchester, UK: Dassault Systèmes Simulia Corp.; 2012, September 25-27.

25. Gorash, Y., Chen, H.. A parametric study on creep-fatigue strength of welded joints using the linear matching method. Int $J$ of Fatigue 2013;Under review, no. IJFATIGUE-D-13-00028, StrathPrints URI: http://strathprints.strath.ac.uk/id/eprint/42607.

26. Welding - Basic welded joint details in steel - Part 1: Pressurized components. No. EN 1708-1:2010 in British Standard. London, UK: The British Standards Institution; 2010.

27. Welding - Fusion-welded joints in steel, nickel, titanium and their alloys - Quality levels for imperfections. No. EN ISO 5817:2007 in British Standard. London, UK: The British Standards Institution; 2007.

28. Pedersen, M.M., Mouritsen, O.O., Hansen, M.R., Andersen, J.G., Wenderby, J.. Comparison of post weld treatment of high strength steel welded joints in medium cycle fatigue. Welding in the World 2010;54(7-8):R208-R217.

29. Skelton, R.P., Gandy, D.. Creep-fatigue damage accumulation and interaction diagram based on metallographic interpretation of mechanisms. Materials at High Temperatures 2008;25(1):27-54.

30. Dowling, N.E.. Mechanical Behavior of Materials: Engineering Methods for Deformation, Fracture, and Fatigue. Upper Saddle River, USA: Pearson Prentice Hall; 3rd ed.; 2007.

31. ABAQUS Scripting User's Manual. Dassault Systèmes Simulia Corp.; Version 6.10 ed.; 2010.

32. Stephens, R.I., Fatemi, A., Stephens, R.R., Fuchs, H.O.. Metal Fatigue in Engineering. New York: John Wiley \& Sons; 2 nd ed.; 2001.

33. Bate, S.K., Hayes, J.-P., Hooton, D.G., Smith, N.G.. Further analyses to validate the R5 volume 2/3 procedure for the assessment of austenitic weldments. Report for BE Generation Ltd no. SA/EIG/11890/R002; Serco Assurance; Warrington, UK; 2005.

34. Dean, D.W.. Recent developments in the R5 procedures for assessing the high temperature response of structures. Materials at High Temperatures 2011;28(2):95-102. 


\section{Nomenclature}

\section{Abbreviations}

EPP Elastic-perfectly-plastic

FEA Finite Element Analysis

FSRF Fatigue strength reduction factor

HAZ Heat-affected zone

LCF Low-cycle fatigue

LMM Linear Matching Method

R-O Ramberg-Osgood

TIG Tungsten-arc inert-gas

\section{Variables, Constants}

$\sigma$

$\Delta \sigma$

$\sigma_{\text {eop }}$

$\varepsilon$

$\dot{\varepsilon}$

$\tilde{\varepsilon}$

$\Delta \varepsilon$

$\omega$

$t$

$\Delta t$

E

$\bar{E}$

$\mu$

$N$

$L$

$A, n, m$

Z

$B, k$

$M$

$\tilde{M}$ stress

stress range

edge-of-plate stress

strain

strain rate

plastic-elastic strains ratio

strain range

damage parameter

time

dwell period

Young's (elasticity) modulus

effective elastic modulus

Poisson's ratio

number of cycles

residual life

primary creep material constants

elastic follow-up factor

creep rupture material constants

bending moment

normalised moment
$\Delta M \quad$ moment range

$P$

$I_{X}$

$w$, thk

$\alpha, \beta$

$\rho$

$R_{3}$

$a$

$\sigma_{\mathrm{y}}$

$B, \beta$

$p_{0}, p_{1}, p_{2}$

$a_{0}, \ldots, a_{3}, b_{0}, \ldots, b_{3}$

$m_{1}, \ldots, m_{8}$

normal pressure

area moment of inertia

width and thickness of plate

angles governing the form of

types 1 and 2 weldments

ratio governing the weld form

radius of weld profile

width of weld throat

yield stress

$\mathrm{R}-\mathrm{O}$ model constants

coefficients for parent material

S-N curve

fitting parameters for $N^{\star}$

fitting parameters for $\tilde{M}_{\max }$

Subscripts, Superscripts

0 corresponding to initial value

cr creep

f fatigue

el elastic

pl plastic

* corresponding to pure fatigue

$\star \quad$ corresponding to creep-fatigue

vM von Mises

eq equivalent

tot total

1c per 1 cycle

lim corresponding to limit load

sh corresponding to shakedown limit

par corresponding to parent material

T3 corresponding to type 3 weldment 